\title{
Clinical implementation of radiobiological measures in treatment planning. Why has it taken so long?
}

\author{
Panayiotis Mavroidis 1, 2 \\ ${ }^{I}$ Cancer Therapy and Research Center, University of Texas Health Science Center, San Antonio, Texas, USA \\ ${ }^{2}$ Karolinska Institutet and Stockholm University, Stockholm, Sweden
}

Received September 29, 2013; Revised September 30, 2013; Accepted September 30, 2013; Published Online October 2, 2013

\section{Editorial}

It has been at least three decades since radiobiological models based on dose volume histograms (DVH) or 3-dimensional dose distributions started appearing in the literature as means to evaluate and compare radiotherapy treatment plans. Since then, many studies have shown that radiobiological treatment plan evaluation provides a closer association of the delivered treatment with the clinical outcome. This is achieved by taking into account the dose-response characteristics of the irradiated targets and normal tissues involved. In radiobiological treatment planning, biological tissue information and physical data have a complementary relation in analyzing dose plans due to the fact that both are incorporated in the mathematical expressions of the radiobiological models.

However, although the necessity of using radiobiological models in radiotherapy has been proven, their clinical implementation until now is almost negligible. Even the biological effective dose (BED) that was introduced in the early 80 s has mainly been used to associate the maximum BED values of certain tissues with the respective risk for complications. And even though it has become a common knowledge that BED is a more accurate descriptor of the biological effectiveness of the applied physical dose, its clinical use is still limited. It is only during the recent years that BED started being used for dose prescription purposes instead of the physical dose or for determining BED-related dose thresholds for normal tissues. Furthermore, although the different treatment planning systems (TPS) have recently

Corresponding author: Panayiotis Mavroidis, $\mathrm{PhD}$; Division of Medical Physics, Department of Radiation Oncology, University of Texas Health Science Center, San Antonio, Texas USA;

Email: mavroidis@uthscsa.edu

Cite this article as:

Mavroidis P.Clinical implementation of radiobiological measures in treatment planning. Why has it taken so long? Int J Cancer Ther Oncol 2013;1(1):01019. DOI: 10.14319/ijcto.0101.9 implemented the calculation of BED values, they still do not provide BED volume histograms or 3-dimenstional BED distributions or iso-BED line charts or the possibility of calculating the composite BED values in treatments composed by multiple phases. Usually, the clinical implementation of a new concept in radiotherapy is the result of the synergetic action between the clinical need and the availability of the relevant tools by the different TPSs.

The facts described above for the BED concept to a large extent hold for the clinical implementation of radiobiological models, namely the models that estimate the tumor control probability (TCP), normal tissue complication probability (NTCP) and complication-free tumor control probability $\left(P_{+}\right)$. These models use radiobiological parameters that describe the dose-response relations of the different tumors and normal tissues. The derivation of the model parameters can be performed by different means such as a maximum likelihood fitting using a cohort of patients for whom the delivered 3-dimensional dose distributions and fractionation scheme as well as the relevant treatment related organ responses have been registered. Although radiobiological models use the complete dosimetric information of a given treatment plan in order to quantify its quality, in clinical practice dose prescription and treatment plan evaluation are based on single doses in the tumor, mean physical doses to the PTV or physical dose-volume points to the tumor and involved organs at risk (OARs), which however take into account a fraction only of the treatment information. Furthermore, the values of those treatment plan quality descriptors are usually characterized by large uncertainty intervals, which however are not taken into account clinically.

The current status of treatment planning practice is the result of the traditional approach of using dosimetric descriptors for quantifying the quality of treatment plans and the fact that the values of these descriptors could be calculated by the TPS, which made their clinical implementation easier. However, all these descriptors are indirectly related 
with treatment plan quality and they do not answer to the fundamental questions of 'what if the likelihood to achieve tumor control' or 'what is the likelihood to have side effects to the OARs' when evaluating a given treatment plan. These answers are provided by the TCP and NTCP measures irrespective of the treatment technique, cancer site, fractionation scheme or clinical protocol applied. So, although radiobiological models convey to a larger extent the clinical insight and the values of their parameters are more accurate compared to those of the descriptors that are used clinically, their clinical implementation is negligible.

There are two main reasons for this. First, there is lack of understanding the theory and the basic concepts behind radiobiological modeling by many radiation oncologists. It can be easily shown that the classes devoted to radiobiological modeling either during the university studies or during the medical residency programs are very limited in number and extent. Second and more important, is the fact that only recently some TPS started offering some tools for performing radiobiological evaluation and comparison of different treatment plans. But still this is not adequate because in order these tools to be used clinically, one has to reach the point of having established radiobiological treatment planning as a clinically implemented procedure.

In order to reach this point, the different TPS should offer to the clinician's tools that will help them organize their patient treatment information and follow-up registrations in order to enable them determine the values of the radiobiological parameters of the models they want to use, based on their own patient data. Appropriate tools should be offered for performing clinical verification of such clinically derived parameter values as well as for performing clinical validation of parameter sets found in the literature. By using those tools, patient datasets consisting of individual tissue response and dose distribution data can be fitted by different radiobiological models and their goodness-of-fit can be evaluated. These are the important elements that are missing for a clinical implementation of radiobiological modeling, the absence of which has led to this long delay in seeing a reasonable progress in this issue.

Radiobiological treatment plan evaluation may allow a fairly accurate prediction of tumor control or normal tissue complications taking into account the variations in patient radiosensitivity. The use of radiobiological modeling is necessary if a clinically relevant quantification of a dose plan is needed.

(Dr. Panayiotis Mavroidis is an editorial board member of International Journal of Cancer Therapy and Oncology) 